\title{
Expression of PD-L1 and presence of CD8-positive $T$ cells in pre-treatment specimens of locally advanced cervical cancer
}

Emeka K Enwere ${ }^{1}$, Elizabeth N Kornaga ${ }^{1}$, Michelle Dean ${ }^{1}$, Theodora A Koulis ${ }^{2}$, Tien Phan $^{2}$, Maria Kalantarian ${ }^{1}$, Martin Köbel ${ }^{3}$, Prafull Ghatage ${ }^{2}$, Anthony M Magliocco ${ }^{4}$, Susan P Lees-Miller ${ }^{5}$ and Corinne M Doll ${ }^{2}$

${ }^{1}$ Translational Laboratories, Tom Baker Cancer Centre, Calgary, AB, Canada; ${ }^{2}$ Department of Oncology, Tom Baker Cancer Centre, Calgary, AB, Canada: ${ }^{3}$ Department of Pathology and Laboratory Medicine, Calgary Laboratory Services, Calgary, AB, Canada; ${ }^{4}$ Department of Anatomic Pathology, H. Lee Moffitt Cancer Center, Tampa, FL, USA and ${ }^{5}$ Department of Biochemistry and Molecular Biology, University of Calgary, Calgary, $A B$, Canada

Several of the cancer immunotherapies under investigation or in clinical use target the programmed death-ligand 1/programmed death-1 (PD-L1/PD-1) signaling axis. PD-L1 expression in tumor samples has been used as a predictive marker for response to these therapeutics, and may also have independent prognostic utility when assessed along with immune cell markers. Our objectives were to assess the expression of PD-L1 in tumor specimens from a uniformly treated patient cohort with locally advanced cervical cancer, and to determine its prognostic significance along with the density of tumor-infiltrating $T$ cells. We identified 120 patients with locally advanced cervical cancer treated with radical chemoradiotherapy, and built tissue microarrays from their formalin-fixed, paraffin-embedded pre-treatment biopsies. We used conventional brightfield and fluorescence immunohistochemistry to detect PD-L1, and quantified protein expression using both manual pathologist scoring and automated software analysis. We also evaluated the effect of PD-L1 expression in tumors, along with the presence and density of intra-tumoral $\mathrm{CD}^{+} \mathrm{T}$ cells, on patient survival outcomes. Approximately $96 \%$ of the tumor samples expressed PD-L1, as determined using quantitative software analysis. Neither expression of PDL1 nor density of $\mathrm{CDB}^{+} \mathrm{T}$ cells was associated with progression-free or overall survival. However, there was a trend towards worse progression-free survival in patients whose tumors expressed PD-L1 but lacked CD8 ${ }^{+}$ $T$ cells (hazard ratio $=0.43(0.18-1.01), P=0.053)$. Nevertheless, the high percentage of cervical cancer tumor samples expressing PD-L1 suggests that anti-PD-L1 or anti-PD-1 therapies are potential treatment options for this patient population.

Modern Pathology (2017) 30, 577-586; doi:10.1038/modpathol.2016.221; published online 6 January 2017

Cervical cancer is one of the most common cancers in women, with a worldwide annual incidence of 500000 cases and 270000 resultant deaths. ${ }^{1}$ A significant proportion of patients present with locally advanced cervical cancer. ${ }^{2}$ The standard treatment for these women involves concurrent cisplatin-based chemotherapy and radiotherapy, the pairing of which represents the most significant advance in cervical cancer treatment for over a

Correspondence: Dr CM Doll, MD, Department of Oncology, Tom Baker Cancer Centre, 1331 29th Street NW, Calgary, AB, Canada T2N 4N2.

E-mail: corinne.doll@ahs.ca

Received 12 August 2016; revised 10 November 2016; accepted 11 November 2016; published online 6 January 2017 decade. Nevertheless, many patients are either resistant to chemoradiotherapy or relapse following treatment, resulting in $30-40 \%$ mortality over 5 years. $^{2,3}$ Many research efforts are consequently devoted to the identification of prognostic or predictive biomarkers that can assist clinical decision-making, and the development of better therapies for management of locally advanced cervical cancer.

The advent of cancer immunotherapy has significantly improved the management of many malignancies in recent years. It involves the use of targeted agents (often monoclonal antibodies) that inhibit immune checkpoint proteins which suppress the adaptive immune system. ${ }^{4}$ Programmed deathligand 1 (PD-L1, also known as B7-H1 or CD274) is 
one such checkpoint protein, and is expressed on the cell surface of many solid tumors. It inhibits immune surveillance by blocking proliferation and activation of $\mathrm{T}$ cells that express the receptor protein, programmed death-1 (PD-1). PD-L1/PD-1 blockade allows cytotoxic $\mathrm{T}$ cells to recognize and destroy tumor cells, while sparing adjacent normal tissue. Antibodies that interfere with the PD-L1/PD-1 interaction have demonstrated significant clinical activity in a range of malignancies including melanoma, lung cancer, and urothelial carcinoma. ${ }^{5-8}$ Furthermore, the observation that human papillomavirus-driven malignancies such as oral and oropharyngeal carcinomas express PD-L1 ${ }^{9-11}$ has led to clinical trials testing the efficacy of PD-L1/PD-1 blocking immunotherapy in these cancers. ${ }^{12,13}$

Whereas PD-L1/PD-1 inhibitor therapy is an area of active clinical research, there is little information on the expression of PD-L1 in cervical cancer. This dearth of information is surprising given that there are at least two active National Cancer Institute clinical trials, NCT01975831 and NCT02628067, which involve the use of PD-L1/PD-1 inhibitors in patients with cervical cancer. Recent papers have addressed PD-L1 expression in cervical cancer as a correlate of human papillomavirus infection ${ }^{14}$ or copy-number gain of the PD-L1 (CD274) genetic locus; ${ }^{15}$ however, there have been few comprehensive assessments of PD-L1 expression in early stage or locally advanced cervical cancer as a predicate to the utility of checkpoint immunotherapy. Moreover, there are presently no data investigating PD-L1 expression and prognostic impact in cervical cancer patients treated with radical chemoradiotherapy. Emerging data in other tumor sites suggest that high levels of PD-L1 in tumor cells may be a negative prognostic marker, ${ }^{16-18}$ particularly in the context of T-cell infiltration into the tumor microenvironment. ${ }^{19}$

The purpose of this study was to determine pretreatment tumor expression of PD-L1 in locally advanced cervical cancer patients treated with radical chemoradiotherapy. We accomplished this goal using a combination of fluorescence and conventional brightfield immunohistochemistry techniques to combine the sensitivity of the former method with the clinical utility of the latter. We also determined the density of intra-tumoral $\mathrm{CD}^{+} \mathrm{T}$ cells, and correlated PD-L1 expression and T-cell density with clinical outcome.

\section{Materials and methods}

\section{Patient Selection and Chart Review}

This study received ethical approval from the local Institutional Research Ethics Board. Patients included in this retrospective study were women diagnosed with cervical cancer of International Federation of Gynecology and Obstetrics stages IB to IVA. They were all treated with radical chemoradiotherapy with curative intent at a single tertiary cancer care institution (Tom Baker Cancer Centre, Calgary, Alberta, Canada) between 1999 and 2008. Clinical, treatment and follow-up details were extracted from patient charts. Pre-treatment staging included examination under anesthesia, cystoscopy, sigmoidoscopy, chest X-ray, abdominal, and pelvic computed tomography. All patients completed chemoradiotherapy within 180 days of diagnosis.

\section{Treatment}

Radiotherapy involved a combination of wholepelvis external beam radiotherapy and brachytherapy (low-dose or high-dose rate). Pelvic external beam radiotherapy was typically administered by means of a four-field technique using high-energy photons. The most common radiotherapy dose and fractionation regimen was $45 \mathrm{~Gy}$, administered in 25 equal fractions over five weeks (one fraction a day, five fractions a week). Cisplatin chemotherapy, administered concurrently with external beam radiotherapy, was administered at a dose of $40 \mathrm{mg} / \mathrm{m}^{2}$. Unless otherwise required, patients were monitored after treatment with routine clinical exams every 3 months for the first year, every 4 months for the second year, and every 6 months for up to 5 years.

\section{Fluorescence Immunohistochemistry}

We constructed tissue microarrays from archival formalin-fixed, paraffin-embedded pre-treatment biopsies, as described previously. ${ }^{20}$ Sections of these tissue microarrays $(4 \mu \mathrm{m})$ were used in fluorescence immunohistochemistry as described previously. ${ }^{21}$ Heat-induced epitope retrieval was performed by incubating slides in a citrate-based $(\mathrm{pH}$ 6.0) target retrieval solution (S1699, Dako, Mississauga, Canada), and heating to $121^{\circ} \mathrm{C}$ for three minutes in a decloaking chamber (Biocare Medical, Concord, CA, USA). Antibodies used were against PD-L1 (rabbit monoclonal, clone E1L3N, 1:2500, Cell Signaling Technology, Danvers, MA, USA) and pan-cytokeratin (guinea pig polyclonal, catalog number BP5069, 1:100, Acris, San Diego, CA, USA). The PD-L1 antibody has been validated previously. ${ }^{22,23}$ Isotype control antibodies were used at concentrations matched to those of the respective primary antibodies. Immunohistochemistry was performed on a Dako Autostainer Link 48; antibodies were diluted with SignalStain protein blocking reagent, and incubated with the tissue samples at room temperature for $30 \mathrm{~min}$. Secondary antibodies were anti-rabbit EnVision+ (K4011, Dako) and an Alexa 488-conjugated anti-guinea pig antibody (A11073, 1:200, Thermo Scientific, Burlington, ON, Canada). PD-L1 staining was visualized with TSAPlus Cy5 signal amplification reagent (Perkin Elmer, Waltham, MA, USA), and nuclei were visualized 
with 4',6-diamidino-2-phenylindole (Thermo Scientific, catalog number D1306). After immunostaining, slides were coverslipped using ProLong Gold anti-fade mounting medium (Thermo Scientific, catalog number P36934), and stored at $4{ }^{\circ} \mathrm{C}$ until scanned.

\section{Brightfield Immunohistochemistry}

Conventional brightfield immunohistochemistry was performed on a Leica BOND autostainer, using Protocol $\mathrm{F}$ and the Polymer Refine detection kit provided by the manufacturer. Slide dewaxing, rehydration, and epitope retrieval were performed on-board; for the latter, we used the ethylene diamine tetraacetate-based Epitope Retrieval Solution 2 (Leica), and slides were heated to $100{ }^{\circ} \mathrm{C}$ for $10 \mathrm{~min}$ (for PD-L1) or $20 \mathrm{~min}$ (for CD8). The PD-L1 antibody was a rabbit monoclonal (clone E1L3N, 1:100, Cell Signaling Technology), and the CD8 antibody was a mouse monoclonal (clone C8/144B, 1:200, Dako). After staining, slides were dehydrated by stepwise three-minute incubations in 70, 85 and $100 \%$ ethanol, followed by two 5-min incubations in xylenes. The slides were coverslipped using Cytoseal XYL (Thermo Scientific) and dried overnight before visualization.

\section{Immunohistochemical Assessment and Scoring}

The density of $\mathrm{CD}^{+}$cells was scored by one pathologist (MK1) within the tumor compartments of the tissue microarray cores. These cores were assigned scores of 0 (no CD8 ${ }^{+}$cells), 1 (low density, 1-2 CD8 ${ }^{+}$cells per core), 2 (moderate density, 3-15 CD ${ }^{+}$cells per core), 3 (high density, $>15$ countable $\mathrm{CD}^{+}$cells per core), or 4 (extreme density, uncountable $\mathrm{CD}^{+}$cells per core). Tissue microarray cores with $<25 \%$ epithelial tumor content were considered uninterpretable.

Brightfield PD-L1 expression was manually assessed by two pathologists (MK1 and MK2) only in tumor cells. Tumor-associated lymphocytes and macrophages expressing PD-L1 were not scored. PD-L1 expression in tumor cells was scored using the H-score with three intensity levels, and the percent of PD-L1 ${ }^{+}$cells estimated in 1\% steps up to $10 \%$, and thereafter $5 \%$ steps. The mean percentage of PD-L1 ${ }^{+}$cells across all available tissue microarray cores for each observer was used for further analyses.

\section{Image Analysis for PD-L1 Protein Expression}

Tissue microarray slides stained by fluorescence immunohistochemistry were digitized with an Aperio Scanscope FL, and multi-channel images were imported into the AQUAnalysis software platform (version 2.4.4.1, Genoptix) ${ }^{21}$ to determine the mean intensity of PD-L1 fluorescent staining intensity within the cytokeratin-positive tumor area.
Tissue microarray samples were not analyzed if fewer than 200 tumor cells were present. The data were reported as tumor AQUA scores (tAQUA, Figure 1).

\section{Image Analysis for Proportion of Tumor Cells Expressing PD-L1}

To determine the percentage of $\mathrm{PD}-\mathrm{L}^{+}{ }^{+}$tumor cells, an algorithm was designed within HALO image analysis software (version 1.94.392, Indica Lab), using the Membrane FL module to identify PD-L1 expressing cells within the cytokeratin-positive tumor area. To verify the accuracy of the detection, a mechanistically different algorithm was designed using the Cytonuclear FL module. Concordance was high between the algorithms for total number of cytokeratin-positive cells detected (Lin's concordance: 0.921 (95\% confidence interval (CI): 0.9080.933)) and percentage of PD-L1-positive cells (Lin's concordance: 0.982 (95\% CI: 0.978-0.985)). Results of the Membrane FL algorithm were used for further statistical analyses.

\section{Statistical Analysis}

PD-L1 expression was dichotomized at the median PD-L1 tAQUA score or HALO score. Wilcoxon's rank sum, Pearson's $\chi^{2}$ and Fisher's exact tests were used where appropriate. Agreement was assessed using Lin's concordance correlation coefficient. ${ }^{24}$ Progression-free survival and overall survival were measured from the date chemoradiotherapy was completed, as previously described. ${ }^{25}$ The Kaplan-Meier method using the log-rank test was used for comparisons between groups at 5 years, and the Cox proportional hazard regression model was employed to determine hazard ratio estimates. These statistical analyses were performed using Stata version 12.0 (StataCorp LP, College Station, TX, USA). Inter-rater concordance was assessed using unweighted Cohen's $\kappa$ analysis in R version 3.2.3, for pairwise comparisons. We used the linear weighted $\kappa$ statistic to assess inter-rater concordance across multiple cut points.

\section{Results}

\section{Patient Clinicopathologic Characteristics}

This study included 120 women with locally advanced cervical cancer (International Federation of Gynecology and Obstetrics stages IB to IVA). The most common histology was squamous cell carcinoma ( $88 \%$, Table 1), and the remainder were adenocarcinomas. There were no associations between PD-L1 expression, as measured with fluorescence immunohistochemistry and AQUAnalysis, and clinicopathologic correlates including age at diagnosis, tumor size, stage, and node status (Table 1). 


\section{Cell Line Controls}
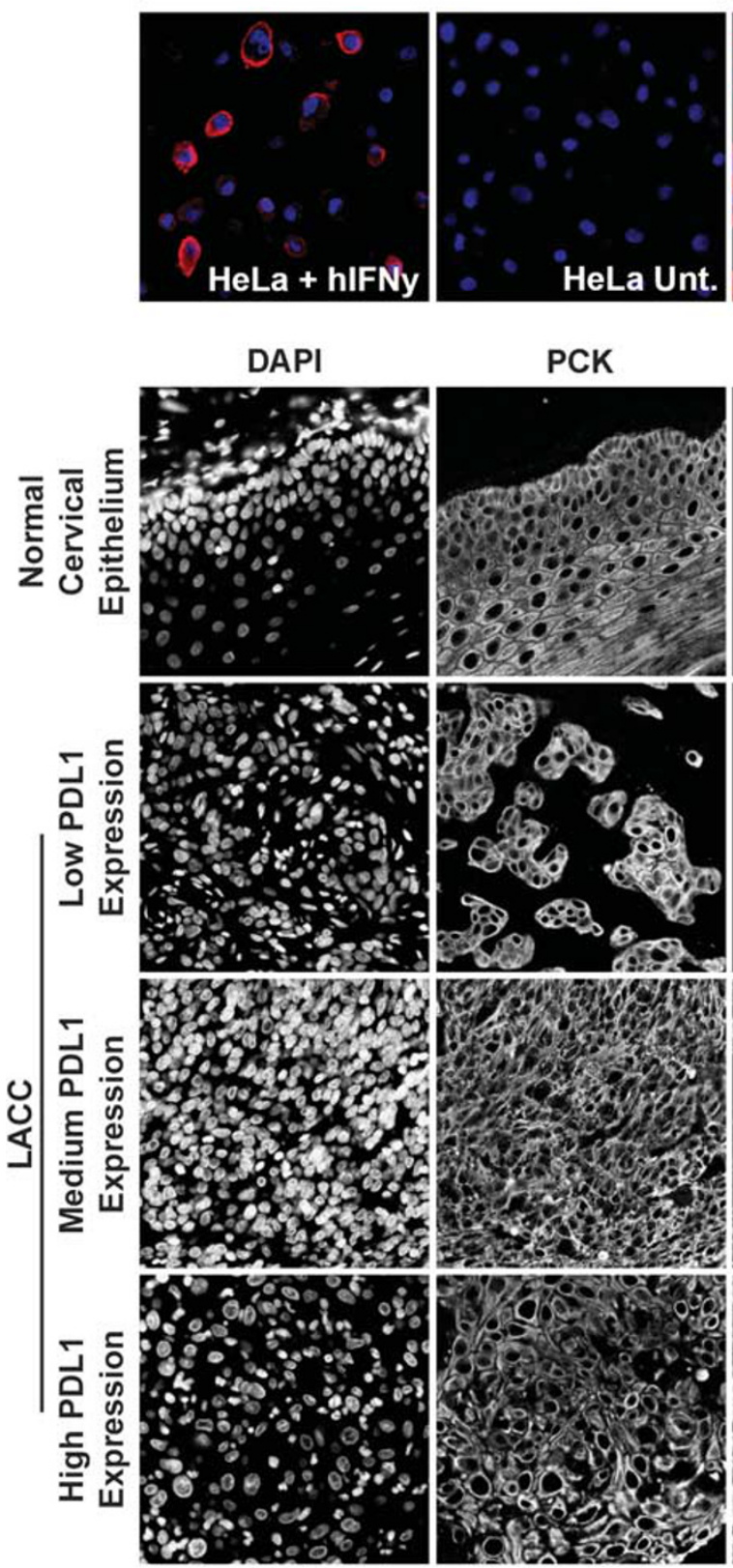

(blue)
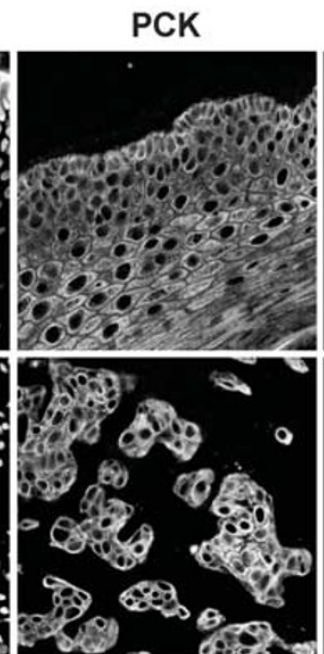

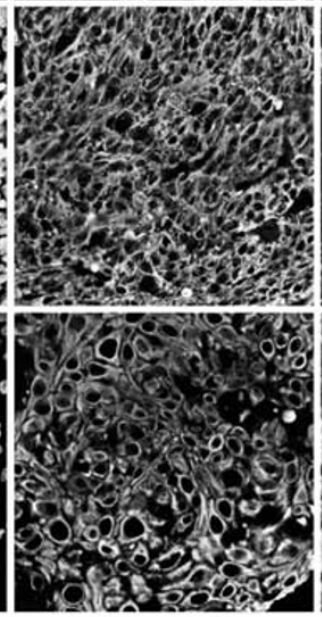

(green)

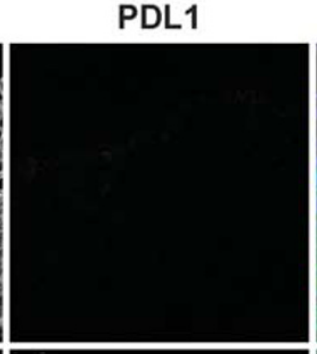

Tissue Controls
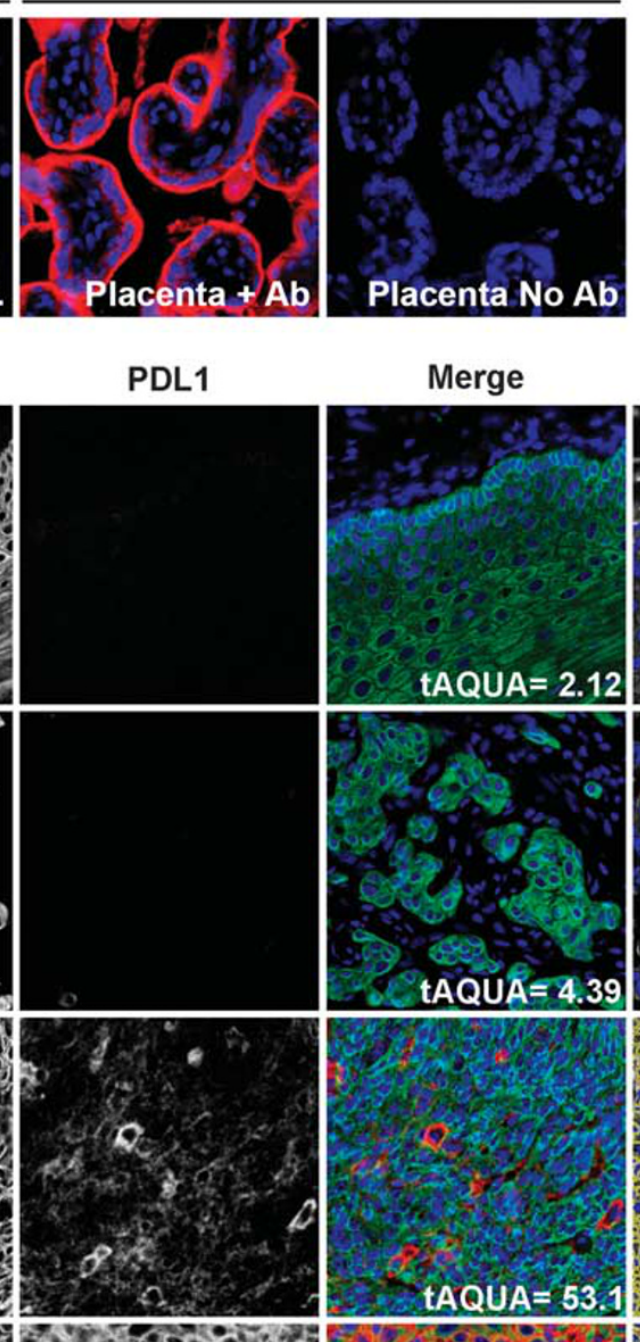

HALO Analysis
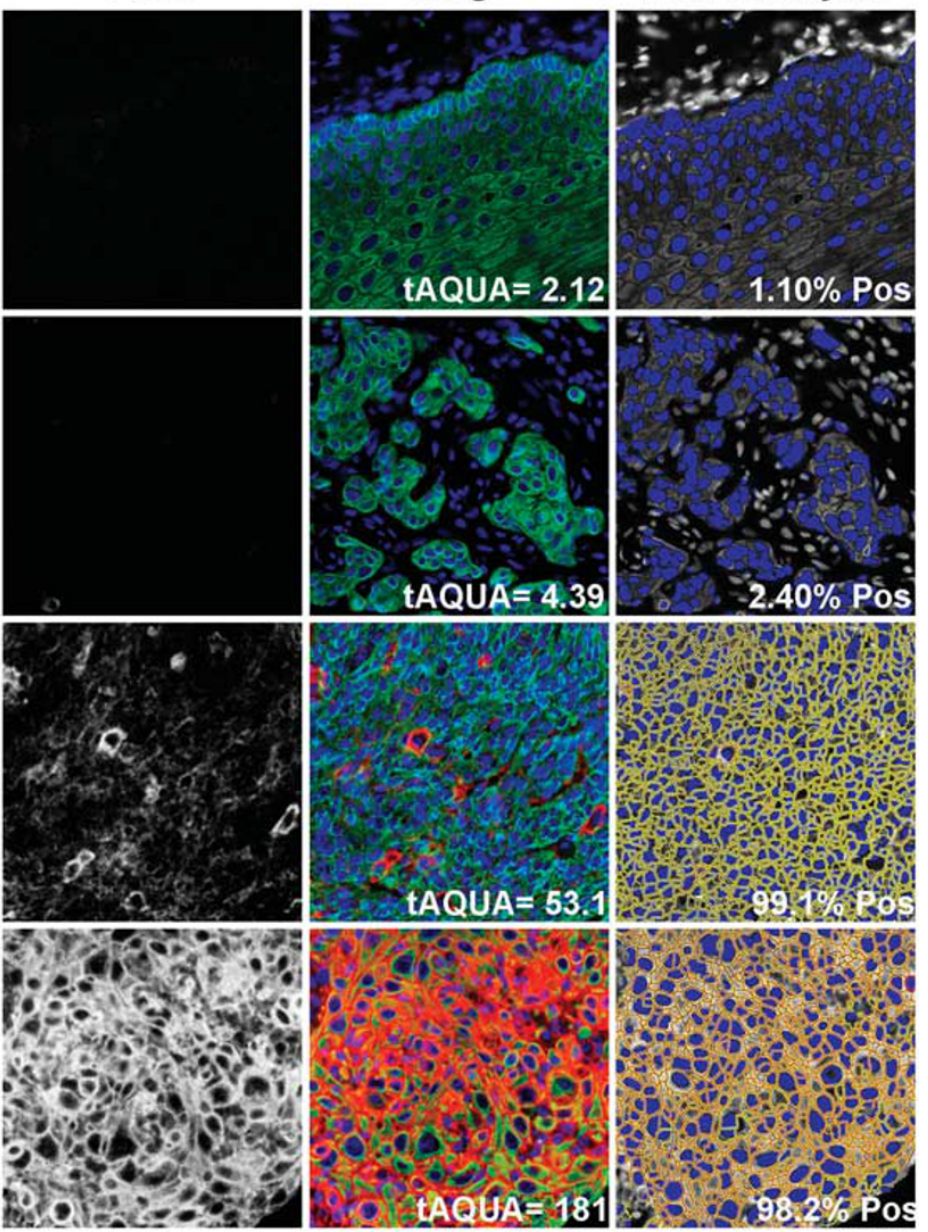

Figure 1 Representative images of fluorescence immunohistochemistry for PD-L1 (programmed death-ligand 1). Samples were stained for PD-L1, as well as cytokeratin to identify tumor regions. Representative tAQUA (tumor AQUA) scores of low, moderate and high PD-L1expressing tumors are indicated. HALO was used to determine the percentage of tumor cells (blue, right column) expressing PD-L1 (yellow outlines, right column).

\section{PD-L1 Expression by Fluorescence} Immunohistochemistry

Representative images of PD-L1 staining in control cells and experimental tissue are shown in Figure 1. Expression patterns of PD-L1 in tumors ranged from diffuse (uniform throughout the tumor) to sporadic. We used HALO image analysis software to determine the percentage of tumor cells that expressed PD-L1.
Approximately $88 \%$ of patient samples were PD-L1positive by the $\geq 1 \%$ cutoff for positivity, ${ }^{26-28}$ and virtually all patient samples $(96 \%$ at the $>0 \%$ cutoff, which represents any positive staining within the tumor) exhibited some degree of PD-L1 staining. Using data from the HALO analysis, we stratified our patient population into groups based on commonly used cut points for PD-L1 expression ${ }^{29}$ (Table 2). The percentage of PD-L1-expressing cells was not 
Table 1 Patient and tumor characteristics

\begin{tabular}{|c|c|c|c|c|c|}
\hline & \multicolumn{2}{|c|}{$\begin{array}{c}\text { Patients, } \\
\mathrm{n}=120\end{array}$} & \multicolumn{3}{|c|}{$\begin{array}{l}\text { Tumor PD-L1 } \\
\text { expression }\end{array}$} \\
\hline & $\mathrm{n}$ & $\%$ & Low & High & P-value \\
\hline \multicolumn{6}{|l|}{ Age } \\
\hline Median & 44 & 48.6 & 60 & 60 & $0.143^{\mathrm{a}}$ \\
\hline Range & $39-49$ & 51.4 & & & \\
\hline \multicolumn{6}{|l|}{ Tumor size $(\mathrm{cm})$} \\
\hline Median & 5.0 & 48.6 & 54 & 54 & $0.6150^{\mathrm{a}}$ \\
\hline Range & $1.5-10$ & 51.4 & & & \\
\hline$\leqslant 5 \mathrm{~cm}$ & 59 & 49.2 & 33 & 26 & $0.214^{\mathrm{b}}$ \\
\hline$>5 \mathrm{~cm}$ & 50 & 41.7 & 22 & 28 & \\
\hline Missing info & 11 & 9.2 & & & \\
\hline \multicolumn{6}{|l|}{ FIGO stage } \\
\hline IB & 24 & 20 & 41 & 38 & $0.564^{\mathrm{b}}$ \\
\hline IIA/B & 55 & 45.8 & 19 & 22 & \\
\hline IIIA/B & 36 & 30 & & & \\
\hline IVA & 5 & 4.2 & & & \\
\hline Low stage (I/II) & 79 & 65.8 & & & \\
\hline High stage (III/IV) & 41 & 34.2 & & & \\
\hline \multicolumn{6}{|l|}{ Histology } \\
\hline SCC & 106 & 88.3 & 55 & 51 & $0.255^{\mathrm{b}}$ \\
\hline Adeno var. & 14 & 11.7 & 5 & 9 & \\
\hline \multicolumn{6}{|l|}{ Nodal status } \\
\hline Negative & 100 & 83.3 & 49 & 51 & $0.807^{\mathrm{C}}$ \\
\hline Positive & 20 & 16.7 & 11 & 9 & \\
\hline
\end{tabular}

Abbreviation: PD-L1, programmed death-ligand 1.

${ }^{\mathrm{a}} P$-value from Wilcoxon.

$\mathrm{b}_{P \text {-value from } \chi^{2} \text { test. }}$

${ }^{\mathrm{C}} P$-value from Fisher's exact test.

Table 2 Number and proportion of patient samples expressing PD-L1 as determined by commonly used cut points, $n=116$

\begin{tabular}{lcc}
\hline$P D-L 1+$ & $\mathrm{n}$ & $\%$ \\
\hline$>0$ & 111 & 95.7 \\
$\geq 1 \%$ & 102 & 87.9 \\
$\geq 5 \%$ & 85 & 73.3 \\
$\geq 10 \%$ & 77 & 66.4 \\
$\geq 25 \%$ & 65 & 56.0 \\
$\geq 50 \%$ & 46 & 39.7 \\
\hline
\end{tabular}

Abbreviation: PD-L1, programmed death-ligand 1.

associated with progression-free survival or overall survival (Figures 2a and b). To determine if level of PD-L1 expression was a prognostic factor, we used AQUAnalysis $^{21}$ to quantify PD-L1 expression as a continuous variable. The tAQUA scores ranged from 1.3 to 163.2 (median $=11.4$ ). Median PD-L1 expression per tumor area (tAQUA) was not associated with progression-free survival (Figure 2c) or overall survival (Figure 2d). Nevertheless, there was a clear correlation between tAQUA scores and HALO-computed percent positivity (Supplementary Figure 1).

\section{The Prognostic Effect of $\mathrm{CD8}^{+} \mathrm{T}$ Cells in the Tumor Environment}

The function of PD-L1 expression in tumors as either a predictive or prognostic biomarker often depends on the presence of $\mathrm{T}$ cells in the tumor microenvironment. ${ }^{30,31}$ To determine if the prognostic value of PD-L1 was affected by the presence of $\mathrm{T}$ cells, we stained separate tissue microarray sections for the T-cell marker CD8. We scored individual tissue microarray cores based on the density of CD8+ cells in the tumor. About 96\% of patient samples contained CD8+ cells (score $>0$, Table 3). To assess the combined prognostic value of PD-L1 expression and the presence of CD8+ cells, we stratified our patient population based on the presence of either criterion. There was no difference in progression-free survival based on CD8 status (CD8+ cells present (CD8+) or absent (CD8-) in tumor samples, Figure 3a), or in PD-L1-expressing patients who did or did not have intra-tumoral CD8 cells (Figure 3b). There were no differences in overall survival based on CD8 alone (Figure 3c; Supplementary Figure 3) or CD8 cells in PD-L1expressing tumors (Figure $3 \mathrm{~d}$ ).

\section{PD-L1 Detection with Brightfield Immunohistochemistry}

To evaluate PD-L1 expression using a more clinically applicable method, we stained tissue microarray sections for PD-L1 using conventional brightfield immunohistochemistry, and scoring was performed independently by two pathologists (MK1 and MK2; Table 4). The E1L3N antibody was validated for brightfield immunohistochemistry using the same IFN $\gamma$-treated cell lines, normal tissue, and patient tumor samples that were used for the fluorescence validation (Supplementary Figure 2). The antibody exhibited similar specificity and dynamic range in brightfield immunohistochemistry as in fluorescence (Supplementary Figure 2; and Dean et al., manuscript in preparation). The correlation between observers' scores as a continuous variable was moderately high (Lin's concordance $=0.832$, 95\% CI: 0.802-0.863), indicating broad congruency in their observations. Inter-rater reliability at each cut point was assessed with Cohen's $\kappa^{32}$ (Table 5). Here, agreement ranged from moderate when the cut point was any positive PD-L1 ${ }^{+}$tumor cells $(\kappa=0.44)$ to good at the $10 \%$ cut point $(\kappa=0.76)$. We also assessed the concordance between individual observers and the fluorescence analysis performed in HALO. It was lowest between MK1 and HALO for any positive cells $(\kappa=0.281)$ and highest between MK2 and HALO at the $25 \%$ cut point $(\kappa=0.703)$. To evaluate interrater concordance across the different cut points, we used the linear weighted $\kappa$ statistic. Concordance was only moderate between pathologists, and between each pathologist and the software analysis (Table 5), ranging from 0.581 to 0.669 . 


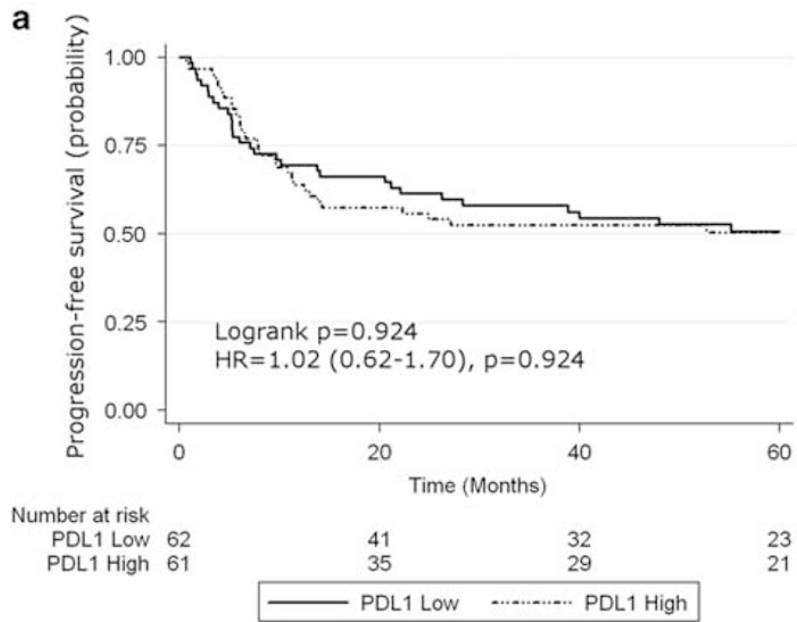

b
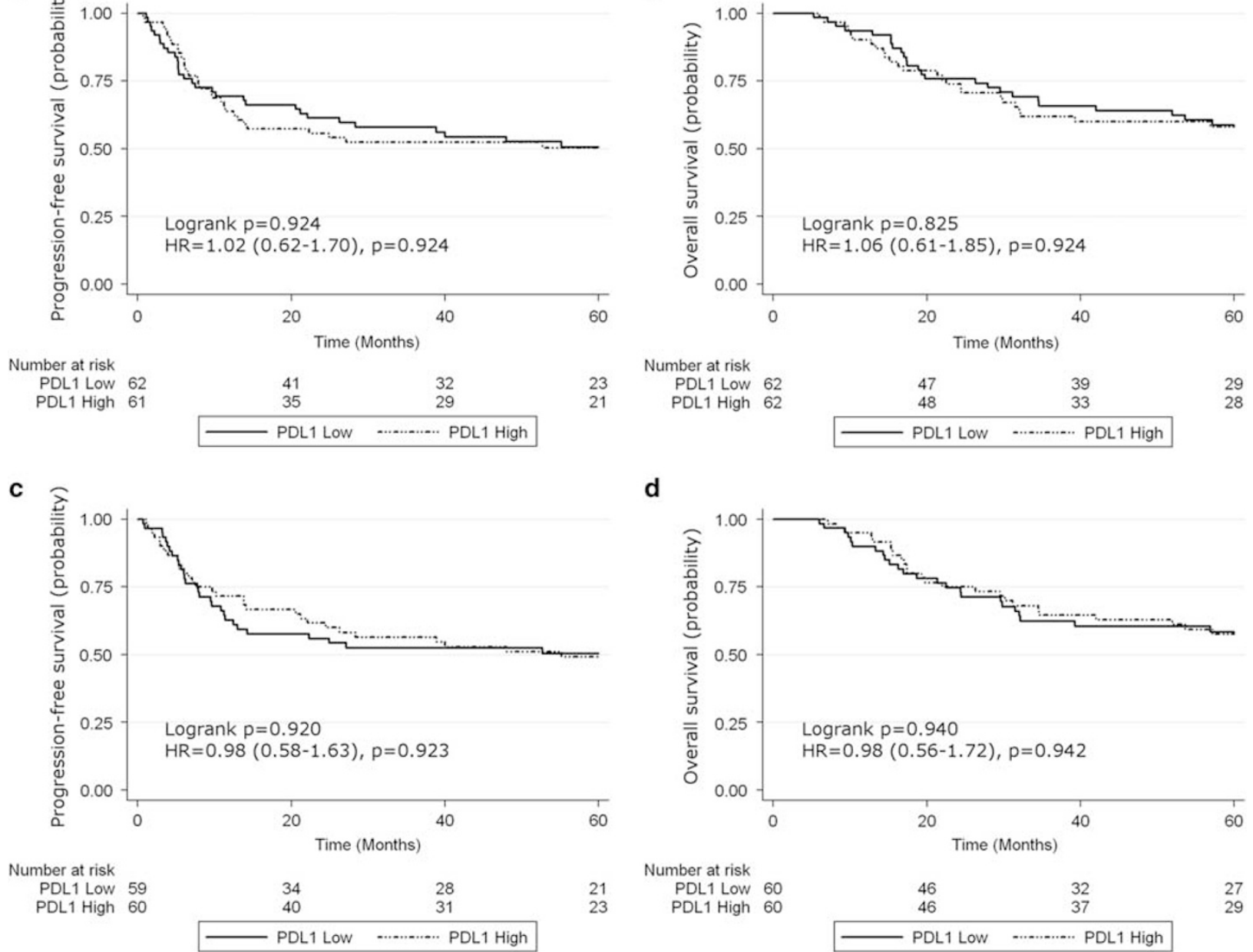

d

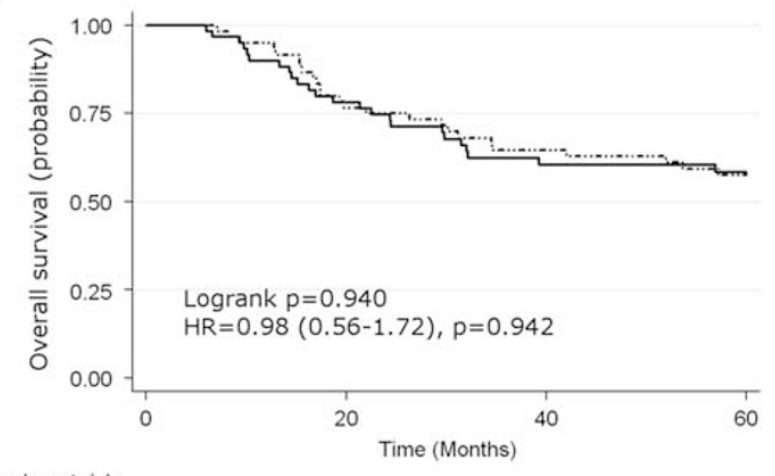

Number at risk
PDL1 Low 60
PDL1 High 60

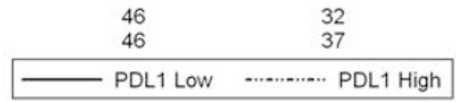

Figure 2 Prognostic value of tumor PD-L1 (programmed death-ligand 1) expression. Kaplan-Meier plots indicating (a) Progression-free survival and (b) overall survival of patients stratified by median percent PD-L1-positive cells in tumor samples; (c) progression-free survival and (d) overall survival of patients stratified by median tAQUA (tumor AQUA) score.

Table 3 Number and proportion of patient samples containing different densities of intra-tumoral $\mathrm{CD}^{+}$cells, $n=111$

\begin{tabular}{lrr}
\hline CD8+ Scores & $\mathrm{n}$ & $\%$ \\
\hline$>0$ & 107 & 96.4 \\
$\geq 1$ & 98 & 88.3 \\
$\geq 2$ & 81 & 73.0 \\
$\geq 3$ & 42 & 37.8 \\
0 & 4 & 3.6
\end{tabular}

\section{Discussion}

The PD-L1 protein is of significant clinical value as a therapeutic target in cancer, and has attracted considerable interest as both a predictive and prognostic marker. In this report, we show that PDL1 is expressed in the vast majority of pre-treatment locally advanced cervical cancer specimens, suggesting that anti-PD-1/PD-L1 therapies could be viable in patients with this malignancy. We also show that, while PD-L1 expression is not an independent prognostic factor for progression-free survival or overall survival, the presence of $\mathrm{CD}^{+}$cells within the tumor microenvironment may provide additional value to PD-L1 expression and warrants further study. Finally, we highlight the difficulties in patient stratification based on PD-L1 staining, especially with the current lack of consistent guidelines for staining methods and cut point definitions.

A limited number of recent papers have also investigated PD-L1 expression in cervical cancer; along with our current study, we believe these papers present separate but collectively useful insights into the biology of checkpoint regulation in cervical cancer. The work by Scott Rodig's group ${ }^{15}$ focused on biopsy specimens, and reported copynumber gains at the $P D-L 1$ gene locus in squamous cell carcinomas in their study population. Another recent publication by Hereen and colleagues ${ }^{33}$ focused on surgically treated early stage cervical cancer patients, and found positive PD-L1 staining (defined as $\geq 5 \%$ ) in $33 \%$ and $40 \%$ of their 2 patient 
a
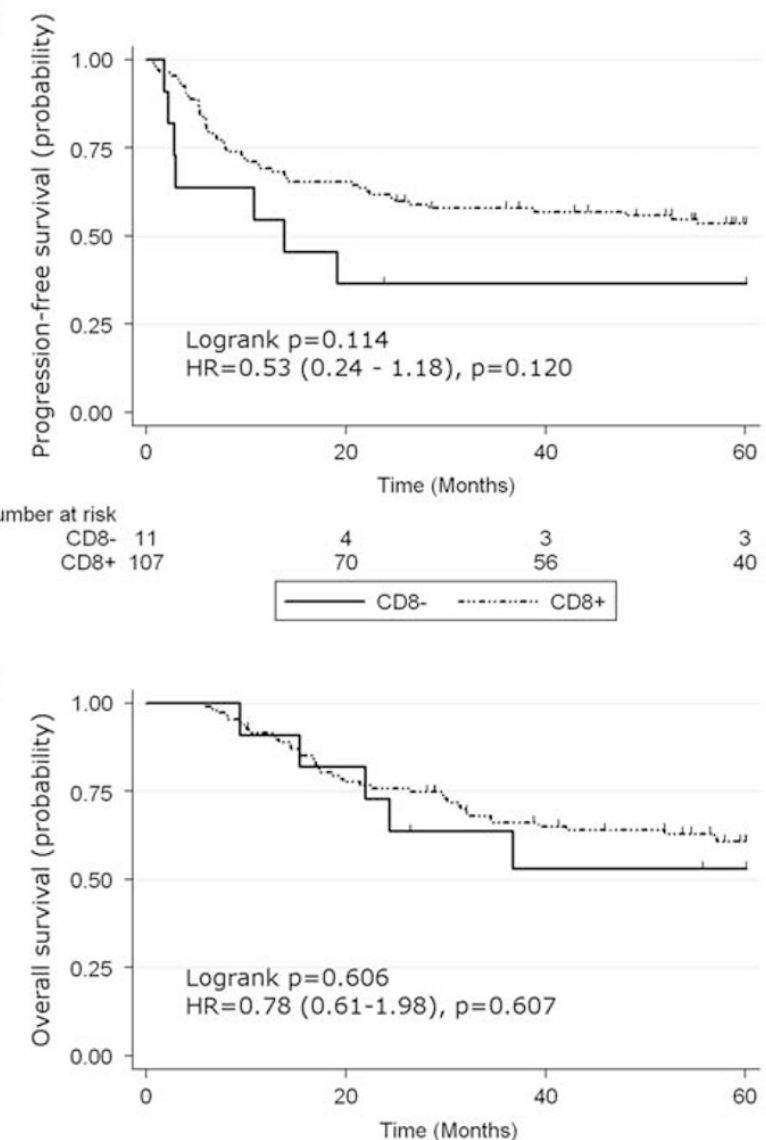

Number at risk

CD8- 11

CD8+ 108 b

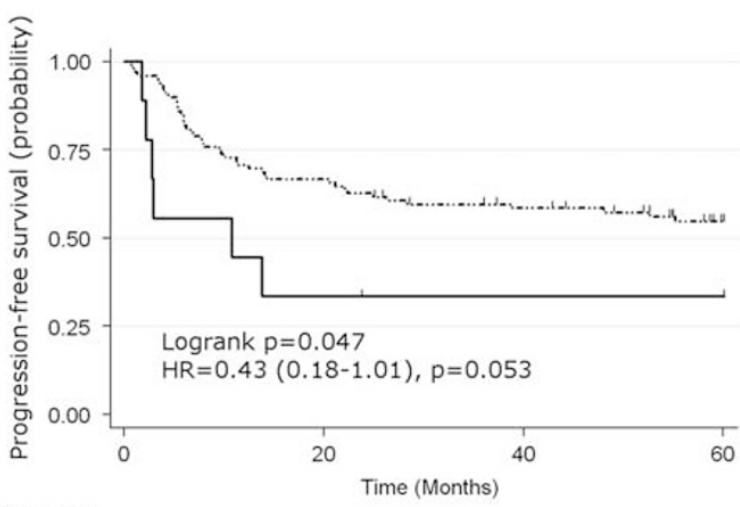

Number at risk

CD8- 9

CD8+ 99

d

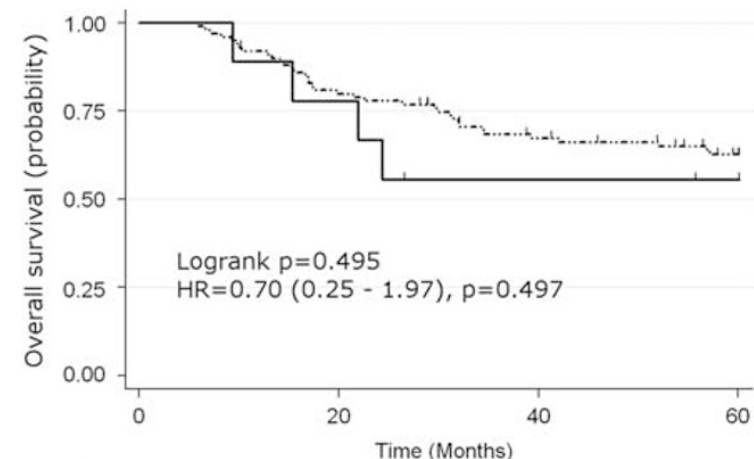

Number at risk

CD8- 9

CD8 +100

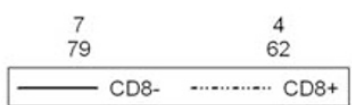

Figure 3 Prognostic value of tumor PD-L1 (programmed death-ligand 1) expression and CD8-positivity. Kaplan-Meier plots indicating (a) progression-free survival of patients stratified by absence (CD8 score 0) or presence (CD8 scores 1-3) of CD8+ cells in tumor samples; (b) progression-free survival of PD-L1-expressing patients, stratified by absence or presence of CD8+ cells; (c) overall survival of patients stratified by absence or presence of CD8+ cells; (d) overall survival of PD-L1-expressing patients stratified by absence or presence of CD8+ cells.

Table 4 Number and proportion of patient samples containing PD-L1-expressing tumor cells as determined by two independent pathologists (MK1, MK2), $n=108$

\begin{tabular}{lrccc}
\hline PD-L1+ & $M K 1(n)$ & $M K 1(\%)$ & $M K 2(n)$ & $M K 2(\%)$ \\
\hline$>0$ & 100 & 92.6 & 89 & 82.4 \\
$\geq 1 \%$ & 98 & 90.7 & 88 & 81.5 \\
$\geq 5 \%$ & 84 & 77.8 & 80 & 74.1 \\
$\geq 10 \%$ & 73 & 67.6 & 69 & 63.9 \\
$\geq 25 \%$ & 61 & 56.5 & 51 & 47.2 \\
$\geq 50 \%$ & 42 & 38.9 & 33 & 30.6 \\
\hline
\end{tabular}

Abbreviation: PD-L1, programmed death-ligand 1.

cohorts. This is lower than the $71 \%$ we observed in this study (Table 2), though the same antibody (clone E1L3N) was used in both cases. Another report ${ }^{14}$ that also focused on biopsy-derived specimens reported a similarly high positivity rate $(80 \%)$ to our study.
Table 5 Inter-rater concordance statistics for PD-L1 detection

\begin{tabular}{|c|c|c|c|c|c|c|}
\hline \multirow{2}{*}{$P D-L 1+$} & \multicolumn{2}{|c|}{$\begin{array}{c}M K 1 \text { vs } \\
M K 2\end{array}$} & \multicolumn{2}{|c|}{$\begin{array}{c}M K 1 \text { vs } \\
H A L O\end{array}$} & \multicolumn{2}{|c|}{$\begin{array}{c}M K 2 \text { vs } \\
H A L O\end{array}$} \\
\hline & $\kappa$ & $\mathrm{n}$ & $\kappa$ & $\mathrm{n}$ & $\kappa$ & $\mathrm{n}$ \\
\hline$>0$ & 0.443 & 118 & 0.281 & 108 & 0.429 & 108 \\
\hline$\geq 1 \%$ & 0.506 & 118 & 0.539 & 108 & 0.369 & 108 \\
\hline$\geq 5 \%$ & 0.658 & 118 & 0.631 & 108 & 0.617 & 108 \\
\hline$\geq 10 \%$ & 0.764 & 118 & 0.603 & 108 & 0.676 & 108 \\
\hline$\geq 25 \%$ & 0.748 & 118 & 0.667 & 108 & 0.703 & 108 \\
\hline$\geq 50 \%$ & 0.731 & 118 & 0.578 & 108 & 0.582 & 108 \\
\hline Weighted (all cut points) & 0.669 & 118 & 0.581 & 108 & 0.610 & 108 \\
\hline
\end{tabular}

Abbreviation: PD-L1, programmed death-ligand 1.

Notably, the patient population in ${ }^{33}$ consisted predominantly of early stage disease (60\% FIGO stage IB1), whereas our own study focused on patients with locally advanced cervical cancer. It is particularly 
important to note that the standard of care differs significantly between early stage cervical cancer and locally advanced cervical cancer; ${ }^{2}$ thus both studies are complementary, but focus on distinct patient populations and treatment groups. The prognostic value of different biomarkers will also vary with these parameters. It is also possible that biologic differences exist between locally advanced vs early stage cervical cancers, which may be indicative of immune factors related to human papillomavirus or other molecular effects. Taken together, the data suggest that the expression of PDL1 may vary in cervical cancer as the disease progresses from early stages to locally advanced cervical cancer.

A number of recent studies, including our own, have highlighted the interplay between PD-L1expressing tumor cells and immune cells within their vicinity. Although our study cohort was relatively small, we noted a distinct trend towards shorter progression-free survival in patients with PD-L1 expressing tumors that lacked CD8+ intra-tumoral cells (Figure 3b). Similar results were observed by Karim and colleagues, ${ }^{34}$ though neither study showed any effect of PD-L1 and intra-tumoral lymphocytes on overall survival. The presence of tumor-infiltrating lymphocytes is generally considered a good prognostic factor in many cancer types; ${ }^{35,36}$ however, these cells are often ineffective due to the immunosuppressive environment deployed by the tumor. ${ }^{37}$ In tumors with a high incidence of somatic mutations, such as cervical cancer, ${ }^{38}$ there is likely an abundance of tumor antigens. Recognition of these antigens by $\mathrm{T}$ cells is subsequently hampered by tumor expression of checkpoint regulators such as PD-L1. The success of PD-1/PD-L1 blockade in hyper-mutated tumor types such as lung cancer, bladder cancer, and melanoma ${ }^{6,7,39}$ is noted particularly in patients with both high PD-L1 expression and high densities of tumor-infiltrating lymphocytes. ${ }^{8,39}$ Given the robust expression of PD-L1, the high density of $\mathrm{CD}^{+}$cells, the human papillomavirus-driven antigenic effect and the high degree of somatic mutations in cervical cancer, we would predict that this malignancy would respond well to PD-1/PD-L1 blockade. Additionally, the influence of human papillomavirus, PD-L1 and immune resistance has been observed in other tumor sites. ${ }^{31}$ Given that human papillomavirus infection is sufficient to initiate genomic instability and malignant transformation in cervical cancer, it is likely a significant factor in the immune response in patients with this disease.

The combination of fluorescence and brightfield immunohistochemistry methodologies allowed for comprehensive assessment of PD-L1 expression in this study; nevertheless, there are shortcomings that could be explored in more detail in future studies. Our patient population of 120 was too small for detailed secondary analyses based on stage or histology. Although PD-L1 expression was only measured within the cytokeratin-positive tumor region in the automated analyses, there is a chance that sporadic non-tumor cells were captured in this region as well. This difficulty also exists with manual review, such that definitive identification of such cells may require further immunohistochemical staining. Several recent publications have described prognostic and predictive value to PD-L1 expression in intra-tumoral lymphocytes and macrophages. ${ }^{14,32-34}$ We have focused instead on PD-L1 expression solely within tumor cells, reasoning that the role of expression in immune cells is more complex and less clearly defined than that of the immunosuppressive role of PD-L1 in the tumor. Similarly, we focused solely on enumeration of CD8+ cells without further characterization of their phenotype, based on the literature that associates lymphocyte infiltration into the tumor with good prognosis in several cancers. ${ }^{35-37}$ Nevertheless, it is likely that a full clinical picture may require greater understanding of the interplay among tumor cells, immune cells, and the stroma, particularly in the context of human papillomavirus infection that is ubiquitous in cervical cancer patients.

The use of both automated and manual scoring methods in this study highlights certain common problems associated with manual PD-L1 scoring as applied in clinical use. The patient stratifications provided by brightfield and fluorescence immunohistochemistry analyses were broadly similar in identifying PD-L1 expression in a majority of cases; however, the discordance of brightfield scores between pathologists was significant. This reflects both the difficulty in identifying occasionally weak membrane staining and the variability in quantification for low thresholds (any positive, 1\%, 5\%). The current FDA-approved immunohistochemistry tests (28-8 pharmDx and 22C3 pharmDx, both by Dako; and SP142 by Ventana) all involve membrane staining of tumor cells at any intensity; ${ }^{38}$ however, there are no calibration tools available, nor any current methodology to reliably determine the minimum acceptable intensity of staining. The absence of these assay validation tools, as well as the inherent heterogeneity of PD-L1 staining ${ }^{40}$ leads to significant discrepancies in patient stratification within and between PD-L1 detection assays. ${ }^{18,39-41}$ In our study, inter-rater concordance was consistently lowest at the 'any positive staining' and 1\% cut points (Table 5), which are currently the most common for the PD-L1 treatments in the clinic. It is worth noting that the PD-L1 assays utilized in the nivolumab and pembrolizumab studies were different, and results cannot be directly compared across studies. Early data from the KEYNOTE-010 trial ${ }^{41}$ indicates that patients with tumors designated as PDL1 weakly positive (PD-L1 1\%-49\%) may also benefit from pembrolizumab treatment. Whether efficacy of anti-PD-L1 therapy varies with PD-L1 tumor expression and whether such a relationship is applicable to all histologies or biomarker-defined 
sub-groups remains unclear. Ongoing studies in various other cancers will likely provide additional insight. Discrepancies also arise from the use of different antibodies against PD-L1. In this study, we used clone E1L3N (Cell Signaling), which has been validated previously. ${ }^{17,18}$ The sensitivity of fluorescence immunohistochemistry, coupled with the use of cytokeratin as a tumor marker, may allow for more consistent semi-automated scoring of PD-L1. Notably, in our study the combination of fluorescence and automated scoring compared well against the manual approaches (Table 5). It is important to note that this work does not constitute a formal comparison of automated and manual scoring of PD-L1; such a comparison would require, among other things, a source of truth against which to calibrate and validate the results of the automated approach. We instead present the automated approach as a demonstration of potential, and as an excellent research tool with which to perform the analyses represented here. Although fluorescence immunohistochemistry is not currently in routine clinical use, we suggest that the combination of fluorescence and image analysis software may allow for increased consistency of performance once calibration standards and guidelines are documented.

In conclusion, our results suggest that PD-L1 is expressed in the vast majority of locally advanced cervical cancer specimens. Although PD-L1 expression was not an independent prognostic factor for progression-free survival or overall survival in our cohort, the analysis of infiltrating immune cells within the tumor milieu may provide further insights on progression-free survival outcome, and requires further exploration. The use of image analysis software to score PD-L1 staining may allow greater consistency than conventional manual scoring, particularly with low cutoffs for patient stratification. Finally, the combination of robust PD-L1 expression, extensive T-cell infiltration, altered immune function from human papillomavirus effect and/or a high degree of somatic mutations indicate that cervical cancers may be excellent candidates for PD-1/PD-L1 blocking immunotherapies.

\section{Acknowledgments}

We are grateful for funding support from Alberta Innovates Health Solutions CRIO and the Alberta Cancer Foundation. We acknowledge Ms. Mie Konno for her assistance in specimen acquisition, preparation, and data entry. AMM has received grant and travel funding from Biotheranostics, travel funding from Definiens and Diacarta, honoraria from Leica and Illumina, and has served as a consultant for Bristol-Myers-Squibb and Ventana.

\section{Disclosure/conflict of interest}

The authors declare no conflict of interest.

\section{References}

1 Ferlay J, Soerjomataram I, Dikshit R, et al. Cancer incidence and mortality worldwide: sources, methods and major patterns in GLOBOCAN 2012. Int J Cancer 2015;136:E359-E386.

2 Minig L, Patrono MG, Romero N, et al. Different strategies of treatment for uterine cervical carcinoma stage IB2-IIB. World J Clin Oncol 2014;5:86-92.

3 Chemoradiotherapy for Cervical Cancer Meta-Analysis C. Reducing uncertainties about the effects of chemoradiotherapy for cervical cancer: a systematic review and meta-analysis of individual patient data from 18 randomized trials. J Clin Oncol 2008;26:5802-5812.

4 Trivedi MS, Hoffner B, Winkelmann JL, et al. Programmed death 1 immune checkpoint inhibitors. Clin Adv Hematol Oncol 2015;13:858-868.

5 Brahmer JR, Tykodi SS, Chow LQ, et al. Safety and activity of anti-PD-L1 antibody in patients with advanced cancer. N Engl J Med 2012;366:2455-2465.

6 Powles T, Eder JP, Fine GD, et al. MPDL3280A (antiPD-L1) treatment leads to clinical activity in metastatic bladder cancer. Nature 2014;515:558-562.

7 Larkin J, Chiarion-Sileni V, Gonzalez R, et al. Combined nivolumab and ipilimumab or monotherapy in untreated melanoma. $N$ Engl J Med 2015;373: 23-34.

8 Rosenberg JE, Hoffman-Censits J, Powles T, et al. Atezolizumab in patients with locally advanced and metastatic urothelial carcinoma who have progressed following treatment with platinum-based chemotherapy: a single-arm, multicentre, phase 2 trial. Lancet 2016;387:1909-1920.

9 Lyford-Pike S, Peng S, Young GD, et al. Evidence for a role of the PD-1:PD-L1 pathway in immune resistance of HPV-associated head and neck squamous cell carcinoma. Cancer Res 2013;73:1733-1741.

10 Ukpo OC, Thorstad WL, Lewis JS Jr. B7-H1 expression model for immune evasion in human papillomavirusrelated oropharyngeal squamous cell carcinoma. Head Neck Pathol 2013;7:113-121.

11 Cunha LL, Marcello MA, Morari EC, et al. Differentiated thyroid carcinomas may elude the immune system by B7H1 upregulation. Endocr Relat Cancer 2013;20:103-110.

12 Ferris RL, Blumenschein G Jr, Fayette J, et al. Nivolumab for Recurrent Squamous-Cell Carcinoma of the Head and Neck. N Engl J Med 2016;375: 1856-1867.

13 Seiwert TY, Burtness B, Mehra R, et al. Safety and clinical activity of pembrolizumab for treatment of recurrent or metastatic squamous cell carcinoma of the head and neck (KEYNOTE-012): an open-label, multicentre, phase 1b trial. Lancet Oncol 2016;17: 956-965.

14 Mezache L, Paniccia B, Nyinawabera A, et al. Enhanced expression of PD L1 in cervical intraepithelial neoplasia and cervical cancers. Mod Pathol 2015;28:1594-1602.

15 Howitt BE, Sun HH, Roemer MG, et al. Genetic basis for PD-L1 expression in squamous cell carcinomas of the cervix and vulva. JAMA Oncol 2016;2:518-522.

16 Gevensleben H, Dietrich D, Golletz C, et al. The immune checkpoint regulator PD-L1 is highly expressed in aggressive primary prostate cancer. Clin Cancer Res 2016;22:1969-1977.

17 Finkelmeier F, Canli O, Tal A, et al. High levels of the soluble programmed death-ligand (sPD-L1) identify 
hepatocellular carcinoma patients with a poor prognosis. Eur J Cancer 2016;59:152-159.

18 Leng C, Li Y, Qin J, et al. Relationship between expression of PD-L1 and PD-L2 on esophageal squamous cell carcinoma and the antitumor effects of CD8(+) T cells. Oncol Rep 2016;35:699-708.

19 Tokito T, Azuma K, Kawahara A, et al. Predictive relevance of PD-L1 expression combined with CD8+ TIL density in stage III non-small cell lung cancer patients receiving concurrent chemoradiotherapy. Eur J Cancer 2016;55:7-14.

20 Doll CM, Aquino-Parsons C, Pintilie M, et al. The significance of tumoral ERCC1 status in patients with locally advanced cervical cancer treated with chemoradiation therapy: a multicenter clinicopathologic analysis. Int J Radiat Oncol Biol Phys 2013;85:721-727.

21 Feng X, Li H, Dean M, et al. Low ATM protein expression in malignant tumor as well as cancerassociated stroma are independent prognostic factors in a retrospective study of early-stage hormone-negative breast cancer. Breast Cancer Res 2015;17:65.

22 Wimberly H, Brown JR, Schalper K, et al. PD-L1 expression correlates with tumor-infiltrating lymphocytes and response to neoadjuvant chemotherapy in breast cancer. Cancer Immunol Res 2015;3:326-332.

23 McLaughlin J, Han G, Schalper KA, et al. Quantitative assessment of the heterogeneity of PD-L1 expression in non-small-cell lung cancer. JAMA Oncol 2016;2:46-54.

24 Lin LI. A concordance correlation coefficient to evaluate reproducibility. Biometrics 1989;45:255-268.

25 McIntyre JB, Nelson GS, Ghatage P, et al. PIK3CA missense mutation is associated with unfavorable outcome in grade 3 endometrioid carcinoma but not in serous endometrial carcinoma. Gynecol Oncol 2014; 132:188-193.

26 Herbst RS, Soria JC, Kowanetz M, et al. Predictive correlates of response to the anti-PD-L1 antibody MPDL3280A in cancer patients. Nature 2014;515: $563-567$.

27 Phillips T, Simmons P, Inzunza HD, et al. Development of an automated PD-L1 immunohistochemistry (IHC) assay for non-small cell lung cancer. Appl Immunohistochem Mol Morphol 2015;23:541-549.

28 Romano E, Romero P. The therapeutic promise of disrupting the PD-1/PD-L1 immune checkpoint in cancer: unleashing the CD8 $\mathrm{T}$ cell mediated anti-tumor activity results in significant, unprecedented clinical efficacy in various solid tumors. J Immunother Cancer 2015;3:15.
29 Patel SP, Kurzrock R. PD-L1 expression as a predictive biomarker in cancer immunotherapy. Mol Cancer Ther 2015;14:847-856.

30 Sabatier R, Finetti P, Mamessier E, et al. Prognostic and predictive value of PDL1 expression in breast cancer. Oncotarget 2015;6:5449-5464.

31 Zou W, Wolchok JD, Chen L. PD-L1 (B7-H1) and PD-1 pathway blockade for cancer therapy: mechanisms, response biomarkers, and combinations. Sci Transl Med 2016;8:328rv4.

32 Landis JR, Koch GG. The measurement of observer agreement for categorical data. Biometrics 1977;33: 159-174.

33 Heeren AM, Punt S, Bleeker MC, et al. Prognostic effect of different PD-L1 expression patterns in squamous cell carcinoma and adenocarcinoma of the cervix. Mod Pathol 2016;29:753-763.

34 Karim R, Jordanova ES, Piersma SJ, et al. Tumorexpressed B7-H1 and B7-DC in relation to PD-1+ T-cell infiltration and survival of patients with cervical carcinoma. Clin Cancer Res 2009;15:6341-6347.

35 Garcia-Teijido P, Cabal ML, Fernandez IP, et al. Tumorinfiltrating lymphocytes in triple negative breast cancer: the future of immune targeting. Clin Med Insights Oncol 2016;10:31-39.

36 Bremnes RM, Busund LT, Kilvaer TL, et al. The role of tumor-infiltrating lymphocytes in development, progression, and prognosis of non-small cell lung cancer. J Thorac Oncol 2016;11:789-800.

37 Piersma SJ. Immunosuppressive tumor microenvironment in cervical cancer patients. Cancer Microenviron 2011:4:361-375.

38 Alexandrov LB, Nik-Zainal S, Wedge DC, et al. Signatures of mutational processes in human cancer. Nature 2013;500:415-421.

39 Fehrenbacher L, Spira A, Ballinger M, et al. Atezolizumab versus docetaxel for patients with previously treated nonsmall-cell lung cancer (POPLAR): a multicentre, openlabel, phase 2 randomised controlled trial. Lancet 2016; 387:1837-1846.

40 Botti G, Scognamiglio G, Cantile M. PD-L1 immunohistochemical detection in tumor cells and tumor microenvironment: main considerations on the use of tissue micro arrays. Int J Mol Sci 2016;17:1046.

41 Herbst RS, Baas P, Kim DW, et al. Pembrolizumab versus docetaxel for previously treated, PD-L1-positive, advanced non-small-cell lung cancer (KEYNOTE-010): a randomised controlled trial. Lancet 2016;387:1540-1550.

Supplementary Information accompanies the paper on Modern Pathology website (http://www.nature.com/ modpathol) 